\title{
The Decision to Make a Movement: Neurophysiological Insights
}

\author{
Michael J. Aminoff and Douglas S. Goodin
}

\begin{abstract}
It is unclear how the brain enables a subject to discriminate between two or more sensory stimuli and to respond appropriately to them. This process must include the ability to detect and identify the stimuli, and to select and initiate an appropriate motor response. With the advent of improved computer technology, this behavior can now be studied in the laboratory, not only by monitoring the input to (stimulus) and output from the brain (response), but also by measuring the associated electrical activity of the brain in order to gain an understanding of how this task is accomplished. In this paper we discuss our work in this area and its relevance to understanding the neural organization of the decision to make a movement in response to a sensory stimulus.
\end{abstract}

\begin{abstract}
RÉSUMÉ: La décision d'exécuter un mouvement: point de vue neurophysiologique. La façon dont le cerveau permet à un sujet de discriminer entre 2 stimuli sensitifs ou plus et de répondre de façon appropriée n'est pas clair. Ce processus doit inclure la capacité de détecter et d'identifier les stimuli, et de sélectionner et d'initier une réponse motrice appropriée. Avec l'avènement d'ordinateurs plus puissants, ce comportement peut maintenant être étudié en laboratoire, non seulement en observant l'influx (stimulus) au cerveau et la réponse, mais aussi en mesurant l'activité électrique cérébrale qui y est associée afin d'accroître notre compréhension de la façon dont cette tâche est accomplie. Dans cet article, nous discutons de notre travail dans ce domaine et de sa pertinence dans la compréhension de l'organisation nerveuse en ce qui concerne la décision d'exécuter un mouvement en réponse à un stimulus sensitif.
\end{abstract}

Can. J. Neurol. Sci. 1997; 24: 181-190

The activities of daily life frequently require that a response is made to one or both of two similar stimuli. A red traffic light, for example, dictates that an oncoming driver apply the brakes of the car, whereas with a green light the driver refrains from braking or presses on the accelerator pedal. Although it is easy to model such behavior in the laboratory, for example by asking subjects to respond differently to each of a random sequence of two tones, the manner in which the sensory stimulus is detected and discriminated (i.e., distinguished from other, similar stimuli) and the appropriate response is selected and executed are unknown. Similarly, the relationship of the processes of sensory discrimination to those that lead to the generation of a motor response is uncertain. The present account summarizes our own work in this area and is not intended to be a general review of the topic.

It is appropriate to provide certain simple definitions for those approaching the subject for the first time. A simple reaction task is one in which a single stimulus is presented, and a single specified response to it is required. This contrasts with a choice reaction task in which different responses are required for each of the different stimuli that are presented. A go/no-go reaction task is one in which a response is required to only one of two or more different stimuli. In this latter task, the subject can simply determine whether the presented stimulus is the one to which a response is required, rather than identifying the stimulus in any more detail. In both the simple and the go/no-go reaction tasks, the response may be pre-selected with certainty, whereas in the choice reaction task the actual response must be selected depending upon the stimulus that is identified. Unlike the simple reaction task, the go/no-go task involves signal identification (at least to the extent of determining whether a response is required).

In evaluating the behavioral responses of subjects in such experimental paradigms, psychologists often assume, either implicitly or explicitly, that the processes of signal detection, signal identification, response selection, and response execution proceed serially, with one stage being completed before the next can proceed. Each of these stages may consist of several substages. For example, feature extraction may be considered a part of signal identification, or response programming as the initial part of response execution. These presumed stages or sub-stages of information processing have then been manipulated experimentally to gain insight to the times required to complete each stage in the decision to make a movement as well as to determine the nature and number of the stages involved in a particular task. The assumption of strictly serial processing may not,

From the University of California, San Francisco School of Medicine, San Francisco, California.

RECEIVED JANUARY 7, 1997. ACCEPTED IN FINAL FORM APRIL 4, 1997

Based on the Canadian Society of Clinical Neurophysiologists Guest Lecture, at the Canadian Congress of Neurological Sciences by Dr. Aminoff, June 1996.

Reprint requests to: Michael J. Aminoff, University of California, San Francisco School of Medicine, 505 Pamassus, Box 0114, San Francisco, California, USA 94143-0114 
however, be valid, and indeed there is a growing interest in the concept of distributed parallel processing by neural networks of the brain in the execution of even the most simple of behavioral activities.

Simple computer programs can be designed to model the steps of signal detection, signal identification, response selection, and response execution to provide insight to the different ways in which the brain may accomplish these activities. For example, each step can be made to proceed serially from the preceding one, as discussed above (Figure 1). Alternatively, signal identification may be overlapped with response selection, so that a quicker response follows input of the stimulus. In yet another program, the probable response may be selected before delivery of any stimulus - if the actual stimulus then matches the predicted one, the response is outputted rapidly, whereas failure of the stimuli to match requires a new response to be selected and executed. This last program fully integrates the processes of signal detection and identification with those of response selection and execution so that the response is executed more quickly than with the other programs. In these last two programs, the involved steps proceed serially but the cognitive processes that are modeled are overlapped and may result from parallel processing.

In considering the function of the brain, it is important to bear in mind that it may not function strictly as a serial or parallel processor; rather, different processes or programs may be used depending upon task or subject requirements - perhaps an integrated program for speed of response to an anticipated stimulus, compared to a serial program when greater accuracy is required or when the nature or timing of the next stimulus cannot easily be predicted by the subject.

\section{Can brain events associated with sensory discrimination and response selection be recorded?}

In our basic experimental paradigm, subjects listen to a pseudorandom sequence of two tones, one of which has a frequency of $1000 \mathrm{~Hz}$ and occurs in most $(86 \%)$ of trials, while the other $(2000 \mathrm{~Hz})$ occurs infrequently (14\% of trials). They are required to attend to the rare stimuli by either counting them silently or making a motor response to one or the other stimuli. Cerebral responses are recorded at the vertex (and other scalp locations) with reference to linked ears. The responses to the two tones are averaged separately from stimulus onset (Figure 2). To the frequent tone a negative potential at approximately $100 \mathrm{msec}$ (N1) is followed by a positive (P2) peak at approximately $200 \mathrm{msec}$. A similar response is also seen to the rare tone, but is followed by a second negativity (N2) followed by a later positivity (P3) at about $300 \mathrm{msec}$. Similar findings, initially reported by Sutton and colleagues, ${ }^{\prime}$ have been replicated by numerous other groups. ${ }^{2-5}$ Such results indicate that the cerebral responses associated with the task of discriminating a rare from a frequent sensory stimulus differ depending upon which stimulus is presented.

It might be questioned whether the responses so recorded depend upon the discrimination process itself or are generated simply because of the different physical characteristics of the stimuli (i.e., the $1000 \mathrm{~Hz}$ and $2000 \mathrm{~Hz}$ tones). This can be answered in two ways. First, when the pitch of the frequent and rare tones is interchanged, ${ }^{6}$ the later $\mathrm{N} 2$ and $\mathrm{P} 3$ components are found in the cerebral response to the rare (now the low-pitched) tone. Second, if the subject ignores the stimuli and instead reads a book, the response to both the frequent and rare stimuli is the same, consisting only of $\mathrm{N} 1$ and $\mathrm{P} 2$ components (Figure 2). The $\mathrm{N} 2$ and P3 (event-related) components of the response to the rare stimulus thus depend entirely upon whether the subject attends to the stimulus train and, as shown by others, ${ }^{7,8}$ upon the relative expectancy of the rare and frequent tones. Indeed, these later event-related components can even be evoked (without the earlier N1 and P2 components) when the anticipated stimulus is unexpectedly omitted. ${ }^{9,10}$ The response recorded when the subject is ignoring the rare tones, by contrast, reflects an obligatory response of the nervous system to the stimulus itself and can be

\begin{tabular}{|c|c|c|c|c|c|c|}
\hline \multirow{4}{*}{ Signal detection } & \multicolumn{2}{|c|}{ Serial Program } & \multicolumn{2}{|c|}{ Partially Serial Program } & \multicolumn{2}{|c|}{ Completely Integrated Program } \\
\hline & Step & Process & Step & Process & Step & Process \\
\hline & 5 & - & 5 & & & \\
\hline & 10 & Input: stimulus & 10 & Input: stimulus & & \\
\hline \multirow[t]{4}{*}{ Signal Identification } & 20 & If stimulus $=A$ & & & 5 & Response $=a^{\prime}$ \\
\hline & 30 & then $S=A$ & & & 10 & Input: stimulus \\
\hline & 40 & If stimulus $=\mathbf{8}$ & 20 & If stimulus $=\mathrm{A}$ & 20 & If stimulus $=\mathbf{A}$ \\
\hline & 50 & then $S=B$ & 30 & then response $=a^{\prime}$ & 30 & then Output: response \\
\hline \multirow[t]{4}{*}{ Response selection } & 60 & If $S=A$ & 40 & If stimulus $=B$ & 40 & If stimulus $=B$ \\
\hline & 70 & then response $=\mathrm{a}^{\prime}$ & 50 & then respons $\theta=b^{\prime}$ & 50 & then response $=b^{\prime}$ \\
\hline & 80 & If $S=B$ & & & 60 & Output: response \\
\hline & 90 & then response $=b^{\prime}$ & & & & \\
\hline Response execution & 100 & Output: response & 60 & Output: response & & \\
\hline $\begin{array}{l}\text { Mean step at which } \\
\text { response occurs }\end{array}$ & 100 & & 60 & & 45 & \\
\hline
\end{tabular}

Figure 1: Simplified computer programs designed to detect an incoming stimulus, identify which of two stimuli was presented, select an appropriate response, and execute it. In the program on the left, these steps are performed serially with each step being completed before the next is begun. In the middle (partially serial) program, the steps of stimulus identification and response selection are overlapped. In the completely integrated program (right) all four of these steps have been overlapped and the mean response latency (i.e., the mean step at which the response is output) is considerably shortened compared to the serial program. 


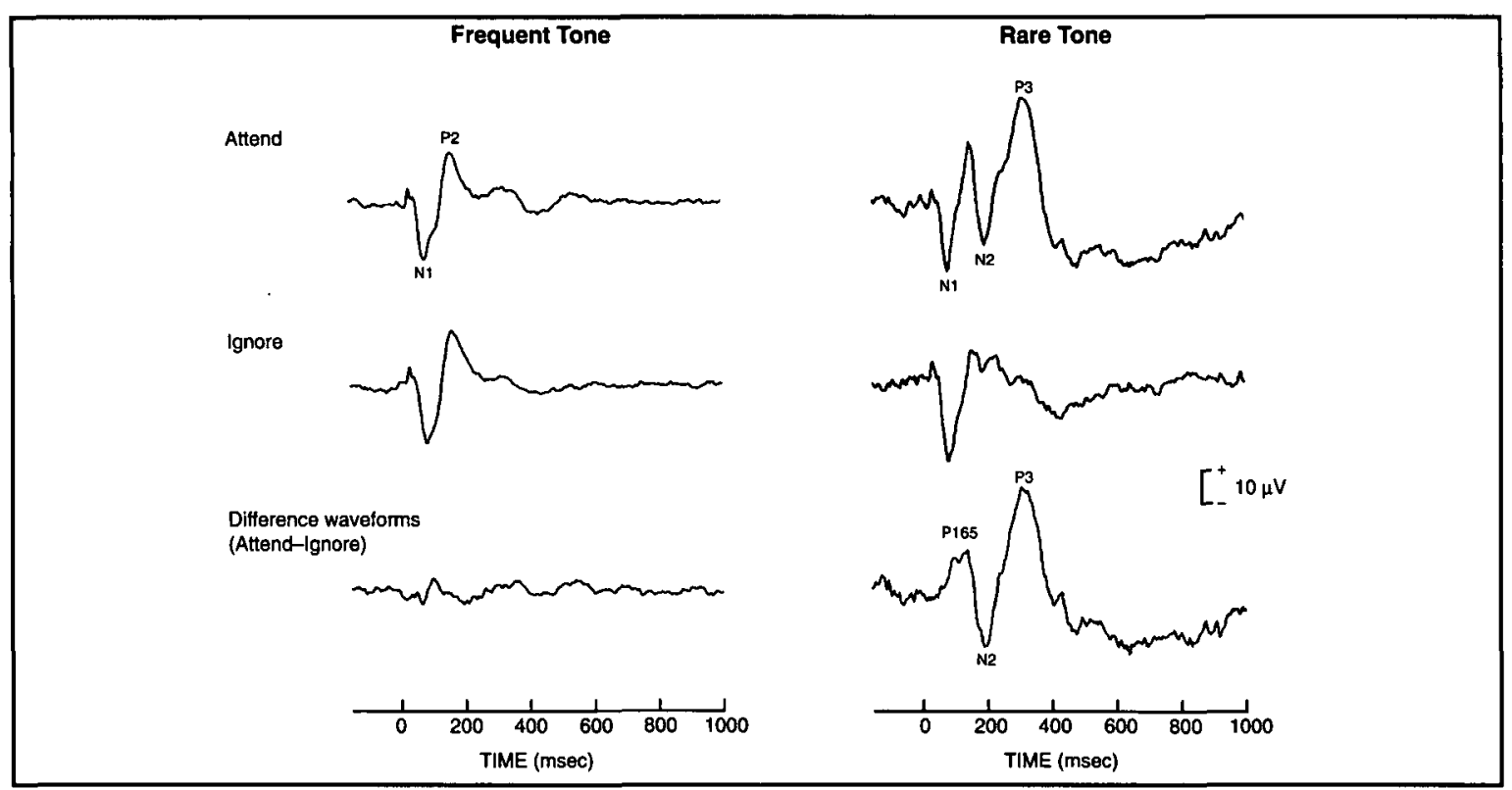

Figure 2: Stimulus-synchronized cerebral responses recorded at $C z$ with reference to linked mastoids following presentation of a frequent (86\% of trials) $1000 \mathrm{~Hz}$ tone (shown on left) and a rare (14\% of trials) $2000 \mathrm{~Hz}$ tone (shown of right). Two attentional conditions are displayed. The top traces (attend) were obtained when the subject mentally counted the occurrence of the rare tones and kept track of the number that had been presented. The middle traces (ignore) were obtained when the subject ignored the tone sequence and read a book. The bottom traces (difference waveforms) were obtained by digital subtraction of the ignore waveforms from the attend waveforms. For the frequent tone response this difference waveform is flat because the cerebral processing of this tone (reflected by the N1-P2 complex) is similar in both attentional conditions and has been subtracted out. For the rare tone response, by contrast, the cerebral activity is different between attentional conditions. In the ignore condition the cerebral potential is characterized by the NI-P2 complex. In the attend condition, the response has several additional potentials so that the difference waveforms reveal the event-related PI65-N2-P3 complex representing the unique cerebral activity following the rare tone in the attend condition.

evoked even when subjects are asleep." It can be subtracted from the response recorded when the subject pays attention to, and discriminates, the rare from the frequent stimulus. This "difference" waveform reflects the brain events specifically associated with the performance of the discrimination task and consists of a positivity having a latency of approximately 165 msec (P165), followed by the N2 and P3 components described earlier (Figure 2). Although these brain events are associated with the discrimination, they do not depend upon which tone is the designated target; rather they depend primarily upon the relative probability of the two tones. ${ }^{6}$

\section{Are the cerebral events related to signal identification pro- cesses, response selection processes, or both?}

To determine whether these cerebral events relate to the stages of information processing described earlier, we modified our basic experiment so that the difficulty in discriminating between rare and frequent stimuli was altered. ${ }^{12}$ The stimuli were of identical pitch $(2000 \mathrm{~Hz})$ but differed in intensity by 20 $\mathrm{dB}$ in one experiment and $3 \mathrm{~dB}$ in another - the distinction between them being more difficult when the difference in intensity was only $3 \mathrm{~dB}$. In each case the physical characteristics of the rare tone were unchanged. The difference waveform (obtained by subtracting the responses when subjects ignored the rare stimuli from those when subjects counted them) demonstrates that all of the event-related components of the cerebral response (P165, N2 and $\mathrm{P} 3$ ) increase in latency relative to stimulus onset when the discrimination is more difficult (Figure 3A), and similar findings have been reported by others. ${ }^{13-17}$ Both the time-locking of these events to stimulus onset and the delay in their occurrence when the discrimination becomes more difficult suggest that the cerebral processes reflected by these eventrelated responses do indeed relate to signal identification. Some investigators have suggested that, under their experimental conditions, such brain events consistently precede and correlate with any motor response required of subjects. ${ }^{4,13,15,16,18}$ Together with observations such as ours, this raises the possibility that these cerebral potentials actually reflect the sensory discrimination process that leads to a response.

To clarify this, we repeated our original experiment but now required subjects to respond by extending the right middle finger whenever a rare tone was detected; this corresponds to a go/no-go reaction task. ${ }^{19}$ The experiment was then repeated with subjects ignoring the rare tone. The cerebral response showed, as before, an N1-P2 response in both the attend and ignore conditions to the frequent tone, and a P165-N2-P3 complex in the difference waveform (either attend minus ignore to the rare tone, or attend-to-rare minus frequent responses). The motor responses were recorded as the unrectified compound muscle action potential (CMAP) in the electromyogram (EMG) using electrodes placed over the motor point of the responding muscle (extensor digitorum communis). Comparison of the onset-latency of the averaged cerebral responses to that of the averaged EMG response showed that the various cerebral responses (apart from the onset of P165) occur too late to be involved in generating the motor response under these go/no-go conditions (Figure 4). It seems, therefore, that although these cerebral potentials reflect some aspect of the signal identification processes, they do not reflect the cerebral events that actually lead to a response, at least under these experimental conditions. It is still possible, however, that the response is triggered at different points of the 


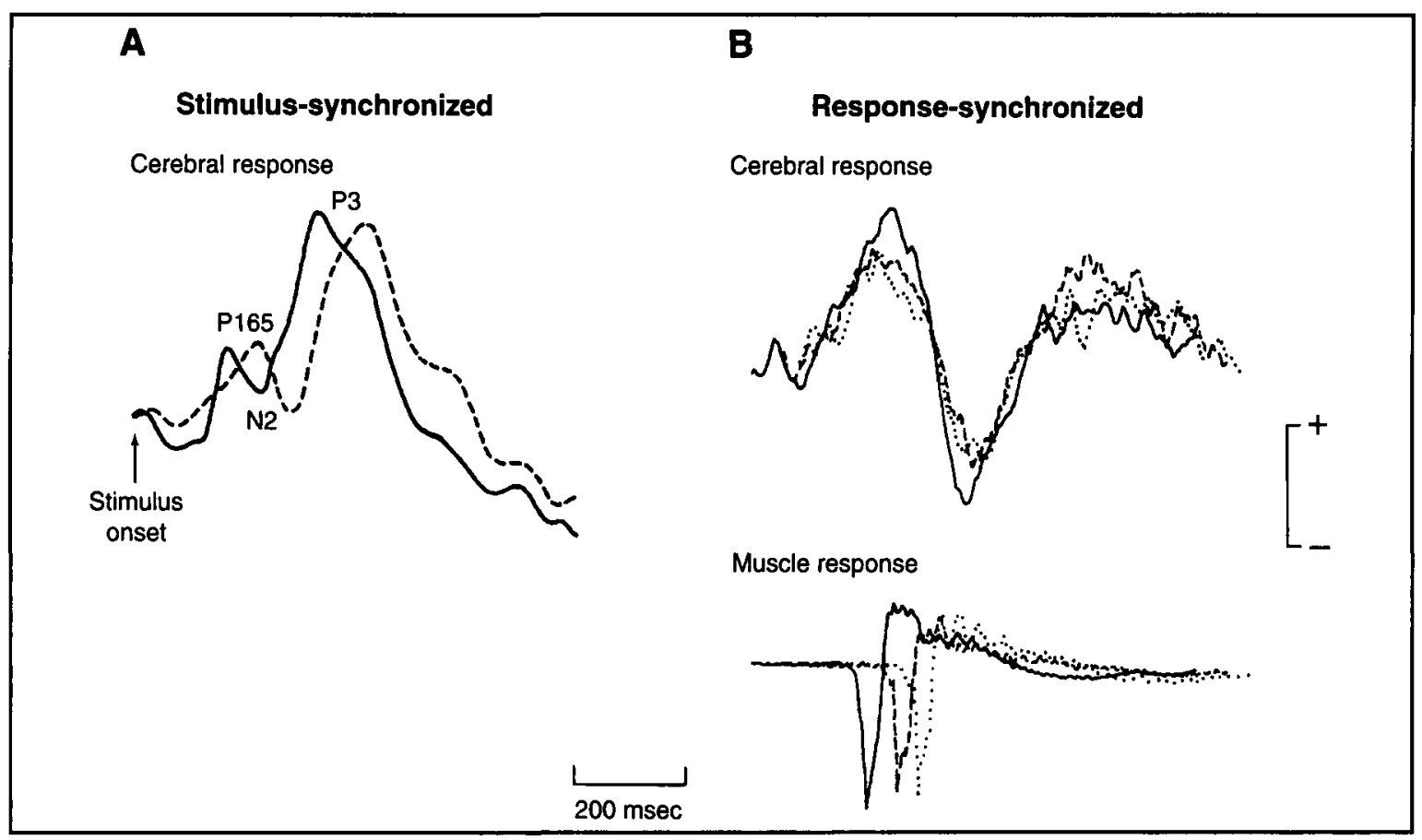

Figure 3: Grand average of the cerebral event-related potentials to rare tones recorded at $C z$ in a group of subjects during tasks involving an easy or difficult sensory discrimination. In A the two tones differed in loudness by either $20 \mathrm{dBHL}$ (easy discrimination - solid line) or by 3 dBHL (difficult discrimination - dashed line), and responses were averaged from stimulus onset (stimulus-synchronized). (Modified from Ref. 12.) Only difference waveforms (attend minus ignore) are shown. All of the event-related potentials (PI65-N2-P3) are delayed relative to stimulus onset in the more difficult task. In B the two tones differed either by the pitch (easiest discrimination-1000 Hz vs. $2000 \mathrm{~Hz}-$ solid line) or by the duration of the tone. The difference in duration was either large (100 msec vs. $50 \mathrm{msec}$ - harder discrimination-dashed line) or small ( $65 \mathrm{msec} v \mathrm{vs} .50 \mathrm{msec}-$ hardest discrimination - dotted line), and responses (both cerebral and muscle) were averaged from response onset (response-synchronized). In each case the rare tone and the required response were identical. Although the only difference between conditions was in the ease of sensory discrimination, there was a progressive delay between the event-related potential (the fused PI65-P3) and the muscle response as the discrimination became harder. In this figure the traces have been aligned by the P165-P3 response to demonstrate more easily the change in coupling between the event-related potential and the response. (Modified from Ref. 21.) The calibration bar indicates $5 \mu \mathrm{V}$ for the cerebral responses and $100 \mu \mathrm{V}$ for the muscle responses.

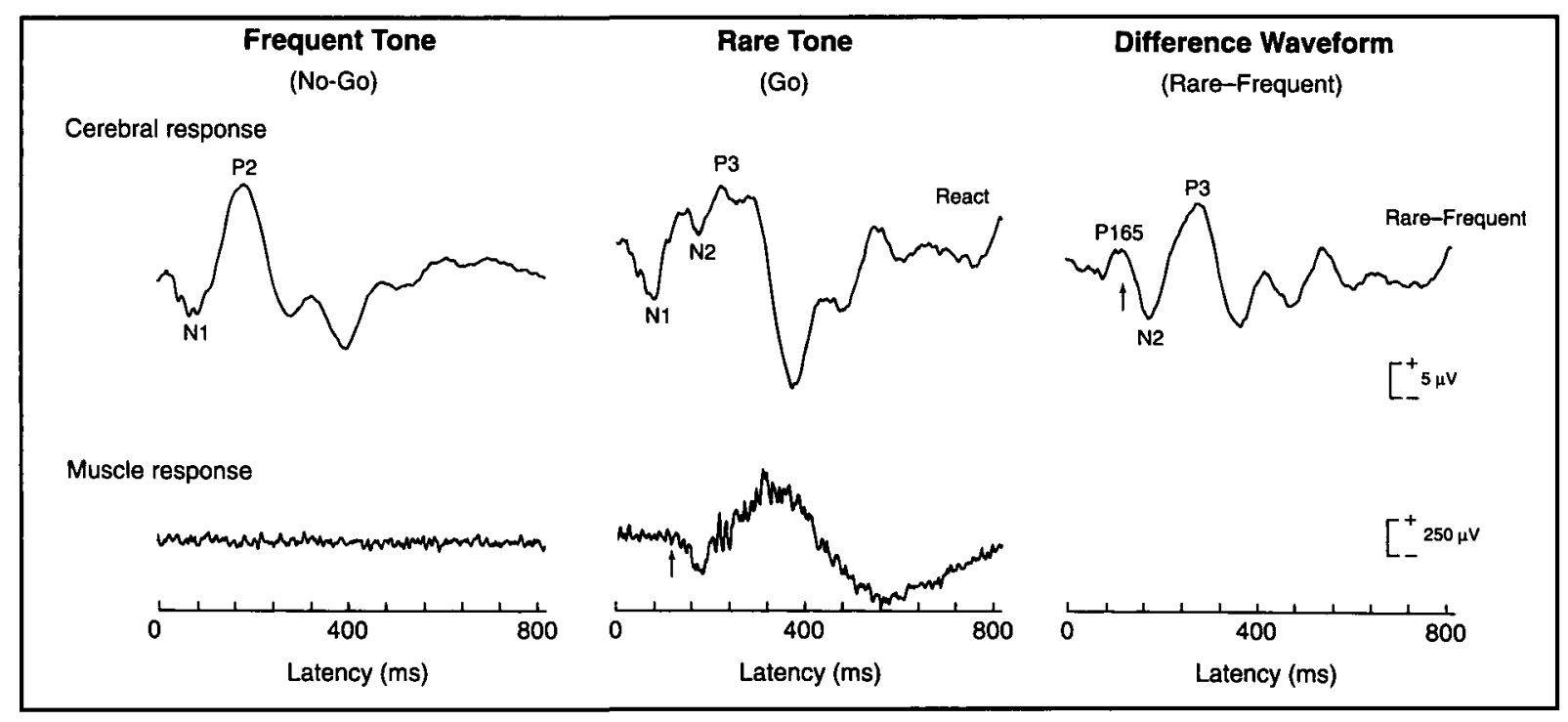

Figure 4: Stimulus-synchronized cerebral and muscle responses to a frequent $(86 \%$ of trials) $1000 \mathrm{~Hz}$ tone and rare (14\% of trials) $2000 \mathrm{~Hz}$ tone in a single subject. Cerebral responses were recorded from $C z$ with reference to linked mastoids and muscle responses from over the motor point of the extensor digitorum communis referenced to the muscle's tendon in this and subsequent figures. Subjects were required to respond to rare tones by extending the middle finger of the right hand as rapidly as possible; no response was required to frequent tones. The difference waveform (rare-frequent) obtained by digital subtraction of the frequent tone waveform from the rare tone waveform is also shown. The onset of the CMAP is identified by a vertical arrow (also shown in the difference waveform) and it can be seen that this onset occurs at about the time of the P165 peak latency. The muscle response to the frequent tone is flat because the subject made no muscle response to this stimulus. (Modified from Ref. 19.) 
signal identification process, depending upon the nature of the task. Alternatively, it remains possible that the response is generated in cerebral regions whose activity is not reflected by the potentials that we recorded, although this would not explain the tight coupling of the response to these potentials.

To examine the possibility that the response is triggered at different points of the signal identification process, we repeated our original experiment with certain modifications..$^{20,21}$ Subjects listened to a pseudorandom sequence of two tones, a rare (14 percent of trials) and frequent one, in three experiments. The physical characteristics of the frequent tone were changed in the different experiments while both the rare tone and the required response were left unchanged. In the first experiment both tones had a duration of $50 \mathrm{msec}$ and were identical, except that the frequent one had a pitch of $1000 \mathrm{~Hz}$ and the rare tone a pitch of $2000 \mathrm{~Hz}$. In the second and third experiments both tones had a pitch of $2000 \mathrm{~Hz}$; the rare tone lasted for $50 \mathrm{msec}$, whereas the frequent tone had a duration of $100 \mathrm{msec}$ in the second experiment and $65 \mathrm{msec}$ in the third. In each experiment, subjects were required to extend the right middle finger in response to the rare tone. Behaviorally (as judged by response-latency), the first discrimination ( $1000 \mathrm{vs.} 2000 \mathrm{~Hz}$ tones) is easier than the second (both tones at $2000 \mathrm{~Hz}$; rare tone $50 \mathrm{msec}$ shorter than frequent tone), and the second is easier than the third (tones at $2000 \mathrm{~Hz}$; rare tone $15 \mathrm{msec}$ shorter than frequent tone). The evoked cerebral potentials were averaged from stimulus onset as before (stimulus-synchronized), but also by back and forward averaging from onset of the CMAP of the responding muscle (response-synchronized). Potentials appearing in the responsesynchronized average must be time-locked, and thus related in some manner, to the response, whereas potentials related to the stimulus but not the response are averaged out. In each case we subtracted the responses to the frequent stimuli from those to the rare stimuli in order to define the event-related potentials that reflect the discrimination process.

Comparison of the stimulus-synchronized difference waveforms and the response-synchronized averages showed that the N2 potential was attenuated in the response-synchronized average, leading to a broad positivity representing a fused (but not attenuated) P165 and P3 (Figure 3B). Alignment of the cerebral potentials in the response-synchronized average by this broad positivity demonstrated that onset of the EMG (motor) response is delayed relative to these cerebral potentials as the discrimination becomes more demanding (Figure 3B).

From these experiments it seems that the P165 and P3 cerebral potentials do not represent strictly sensory or motor events, but rather are closely coupled (i.e., time-locked) to both stimulus and response as judged by the comparable amplitude of these potentials in all conditions (Figure 3B). Nevertheless, the nature of this coupling varies with task complexity: with a more complex task, not only are these cerebral potentials delayed relative to signal onset but the interval between them and the response also increases. The $\mathrm{N} 2$, by contrast, seems more closely coupled to sensory events and was attenuated as noise in the response-synchronized averages. On the basis of these studies, therefore, sensory discrimination, response selection, and response production appear to be overlapping processes, their precise relationship varying depending upon the task and the difficulty of the discrimination.

\section{Are the relationships between cerebral potentials and the response altered when subjects respond differently to each of two stimuli?}

The experiments discussed above were concerned with the potentials recorded when a subject responds to one of two stimuli. What happens when subjects are required to respond differently to each of two stimuli? To examine this, we again presented subjects with a pseudorandom sequence of two tones, and required them to respond to the frequent tone (which occurred in 86 percent of trials) by extending the left middle finger and to the rare tone by extending the right one. ${ }^{22}$ The experiment was done in three different conditions. In one, a choice reaction paradigm, subjects had to respond to whichever stimulus was presented. In a second, a go/no-go task, subjects responded with the left middle finger to the frequent tones and did not respond to the rare tones or, conversely, responded to rare tones by extending the right middle finger and made no response to the frequent tones. In the third condition, a simple reaction task, subjects were presented with only one type of tone (rare or frequent) and had to respond appropriately. In the choice condition, it was clear from analyzing the speed of response to the frequent tones that subjects could be divided into fast responders (mean response latency of approximately $90 \mathrm{msec}$ ) or slow responders (mean response latency of approximately $180 \mathrm{msec}$ ). Fast responders to the frequent tone were also fast responders to the rare tone, and similarly slow responders to the frequent tone were also slow responders to the rare tone (Figure 5). Mean onset latency of the fast response to rare tones was the same as mean onset latency for the slow response to frequent tones. Similar findings were obtained in the go/no-go condition, with an apparent division between fast and slow responders. In the simple reaction task, all subjects were fast responders. These findings suggest that the response to a frequent tone occurs at one of two decision points, but not between them. These results also suggest that the point at which an individual subject responds during the stimulus evaluation process reflects a "strategy" adopted by the subject and that this strategy can be changed between trials. These conclusions are further supported by our findings in four subjects who were fast responders in one trial and slow responders in another; in each case, onset latency of the response showed a step change of $90-100$ msec between trials.

The response to frequent tones was always faster than to rare tones, suggesting that the response to rare tones takes longer because of a bias due to different expectancies. The response latency of the fast-response to the frequent tone was the same in all three conditions (choice, go/no-go, and simple), suggesting that, at least for the fast response, this bias is introduced prior to stimulus-onset. Fast responders to this tone make the determination to respond at the first of the two decision points discussed above regardless of task requirements. Thus, there seems to be a two-step discrimination process - the first to determine whether the tone is frequent or not-frequent and the second to determine whether it is rare or not-rare. For fast responders, the fast response to the frequent tone is made at the first discrimination point and the response to the rare tone is made at the second. This organization is similar to a positive-check model in which a response is made after each stimulus is positively identified. ${ }^{23}$ For slow responders, by contrast, the response to the frequent 


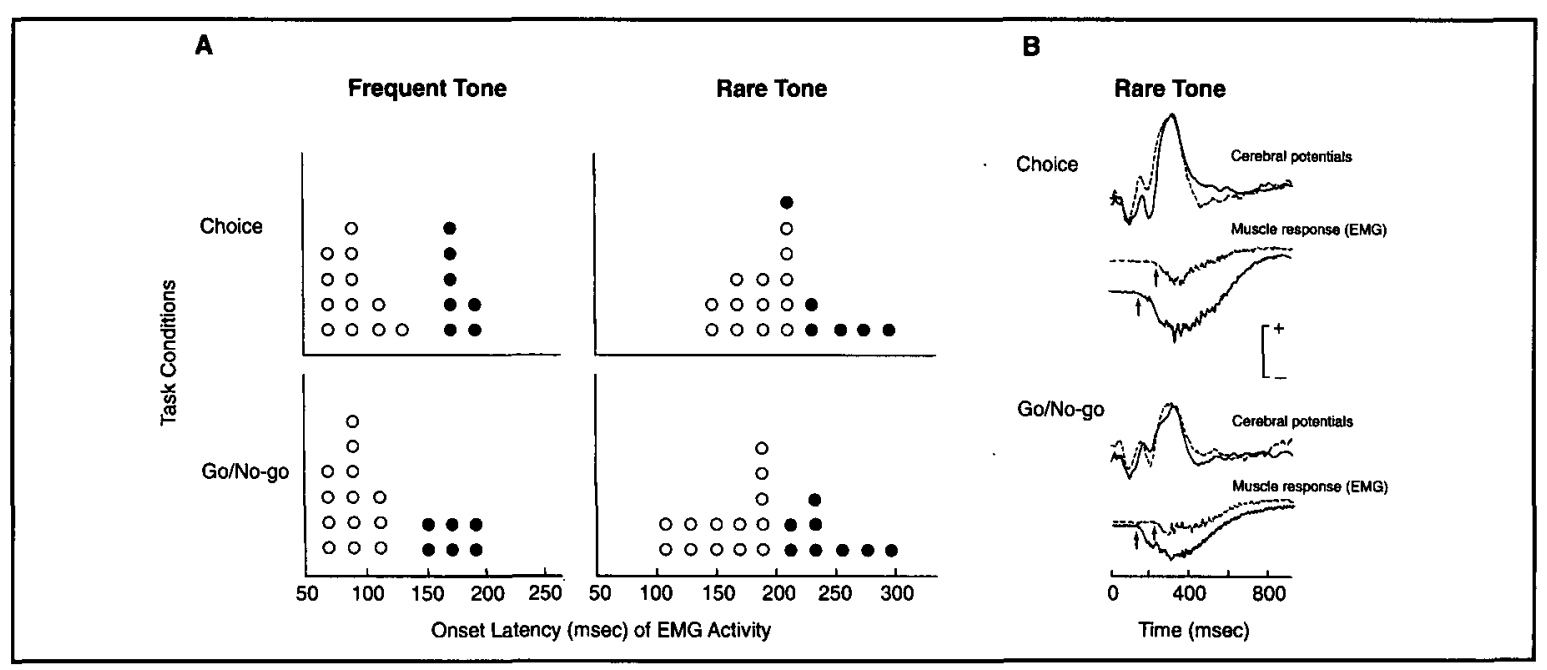

Figure 5: Responses of subjects presented with a frequent ( $86 \%$ of trials) $1000 \mathrm{~Hz}$ tone or a rare (14\% of trials) $2000 \mathrm{~Hz}$ tone. In the Choice condition, subjects responded to the rare stimulus by extending the right middle finger and to the frequent tone by extending the left middle finger. In the Go/No-go condition, subjects responded to either the rare or frequent tone but not both. On the left $(\boldsymbol{A})$ are plotted the onset latencies of the CMAP in the different subjects in the different experimental conditions. The open circles represent fast responders and the filled circles represent slow responders. On the right $(\boldsymbol{B})$, the grand average cerebral and muscle responses to the rare tones in both conditions are shown. Responses from fast responders (solid lines) and slow responders (dashed lines) are shown separately. Vertical arrows indicate the onset of the CMAP in the different conditions. The calibration bar at the right represents $10 \mu \mathrm{V}$ for the cerebral responses and $100 \mu \mathrm{V}$ for the muscle responses. (Modified from Ref. 22.$)$

tone is withheld until the second discrimination point, and the response to the rare tone is delayed even further, presumably due to a response-bias as discussed above.

It is important to note that, despite different response strategies, the cerebral potentials in fast and slow responders were similar in amplitude and latency, suggesting that there were no differences in stimulus-synchronized cerebral processing between them, at least in so far as this is reflected by the potentials that we recorded (Figure 5). Such an organization corresponds to the integrated model of information processing discussed earlier, with a variable coupling of the response to the stimulus evaluation processes depending upon the response strategy adopted by the subject. By contrast, the relationship between these cerebral potentials and the motor response varies in fast and slow responders as demonstrated in the responsesynchronized averages. ${ }^{22}$ In the fast responders in all three conditions, there was a similar coupling of the response to the frequent tone and the P2 cerebral potential. Although the slow responders also show a coupling to this component, it was less tight as reflected by cerebral potentials that were lower in amplitude and longer in duration than those for fast responders; they also occurred earlier relative to the motor response.

\section{Is the discrimination process altered when subjects distin- guish between and respond to each of three stimuli?}

As noted above, the faster responses to the frequent tone are similar (both in mean onset latency and in their coupling to the P2 potential) under all response conditions, suggesting that these subjects prepare to respond to this stimulus in anticipation of its occurrence. Our results also suggest that stimuli are discriminated in the order of their expectancy. To examine this further, we undertook additional studies to determine whether three different tones are also discriminated in order of expectancy and whether slow responders await the results of the complete discrimination before generating a response. ${ }^{24}$ The experiments were somewhat more complicated than those already described.
In brief, subjects were presented with a pseudorandom sequence of three different tones. A frequent tone $(1000 \mathrm{~Hz})$ occurred in 78 percent of trials, a second or "uncommon" tone $(2000 \mathrm{~Hz})$ in 18 percent of trials, and the third or rare tone occurred (4000 $\mathrm{Hz}$ ) in 4 percent of trials. These percentages were chosen so that the ratio of the uncommon to frequent tones was the same as that of the rare to uncommon tones. In one series of experiments, subjects responded with the middle finger of both hands when frequent tones were presented, with the right hand to uncommon tones, and the left hand to rare tones. In a second series, they again responded with both hands to the frequent tones, but with the left hand to uncommon tones, and the right hand to rare tones. Responses were averaged separately for the different response outcomes and conditions (i.e., correct responses and errors to each tone).

In the stimulus-synchronized averages, the cerebral potentials associated with correct responses to the uncommon or rare tones looked similar both to each other and to the rare tone response in the two-choice task described earlier (Figure 2). The cerebral responses to the frequent tone were also similar to those described earlier; the difference waveforms (rare or uncommon tone responses minus frequent tone responses) showed the eventrelated potentials described previously. In addition, the amplitude, latency, and configuration of these event-related potentials were similar for both the uncommon and rare tones, suggesting that either tone evoked substantially similar brain activity. In the response-synchronized averages recorded at the vertex, a slow negative shift in potential representing the late phase of the socalled readiness potential, was associated with each of the three stimuli and was followed by a positive-negative complex (Figure 6 ). When this readiness potential was elicited by the frequent tones, it began prior to stimulus onset, suggesting again that subjects were preparing to respond to this tone in advance of stimulus onset. ${ }^{25}$ (In other experiments involving a simple reaction task, ${ }^{26}$ we found that when subjects were unable to predict the timing of the next stimulus, such anticipatory cerebral processing 
did not occur.) Similar to the two-choice task, the response to the frequent tone was coupled to the P2 cerebral potential whereas the response to the uncommon and rare tones was coupled to the P3 potential (Figure 6). Subjects were again divided into fast and slow responders to the frequent tone, and this predicted their speed of response to the uncommon, but not the rare tone. Latency of response to the rare tones $(412 \mathrm{msec}$ ) was intermediate between the latency for the fast and slow responses to the uncommon stimulus (393 $\mathrm{msec}$ and $453 \mathrm{msec}$ respectively).

When the cerebral potentials recorded over the two sides of the head were examined, the correct response to the uncommon tone was preceded by a correctly lateralized readiness potential, i.e., a slow negative shift that was maximal contralateral to the hand that was moved (Figure 7). Similarly, the lateralized readiness potential to the frequent tone was flat as would be anticipated if, prior to stimulus onset, subjects were preparing to respond with both hands as required. Surprisingly, however, the lateralized potential that preceded a correct motor response to rare stimuli was inappropriately lateralized to the ipsilateral hemisphere, as if subjects were preparing to respond to the uncommon stimulus (Figure 7). Nevertheless, the response to the rare tones was both rapid and accurate.

These findings suggest that, at least for these experimental conditions, the processes of stimulus evaluation and response execution are continuously integrated. The response system is apparently engaged before the occurrence of an anticipated stimulus and then is modified throughout the stimulus evaluation process. A rapid and correct response is thus made despite preparations to generate a different response based on incorrect anticipation of the stimulus.

\section{How does the nature of the response influence the discrimi- nation process?}

In further experiments, we altered the compatibility of the response with the rare stimulus elicited in a visual choice reaction task. ${ }^{27} \mathrm{~A}$ series of seven four-letter words was used for stimulation, six of which were designated as non-targets and occurred in 86 percent of trials, whereas the target was presented in 14 percent of trials. Subjects responded to the non-target stimuli by extending the middle finger of both hands, and to the target stimulus by extending the middle finger of the hand either on the same (compatible task) or opposite (non-compatible task) side as the stimulus. The non-compatible task increased the response time, without altering the amplitude, latency, or configuration of the cerebral responses that we recorded (Figure 8 ). Thus, the change in response time appeared to relate to triggering of the response at different times in the stimulus evaluation process but did not produce any uncoupling of the response from such processes as might have been anticipated if non-compatibility affected only the response system.

Examination of the time-locking between the different cerebral potentials and the response indicated that certain cerebral events, such as the lateralized sensory potential to a visual stimulus (P100), were markedly attenuated (i.e., broadened and flattened) in the response-synchronized averages for both rare and frequent stimuli despite the preservation of other potentials with latency between 100 and $200 \mathrm{msec}$, such as the P165 (Figure 8). A similar attenuation was seen for the error negativity discussed below (Figures 9 and 10). These observations indicate that the $\mathrm{P} 2$ and the event-related potentials are specifically related to the generation of the response and that their preservation in the

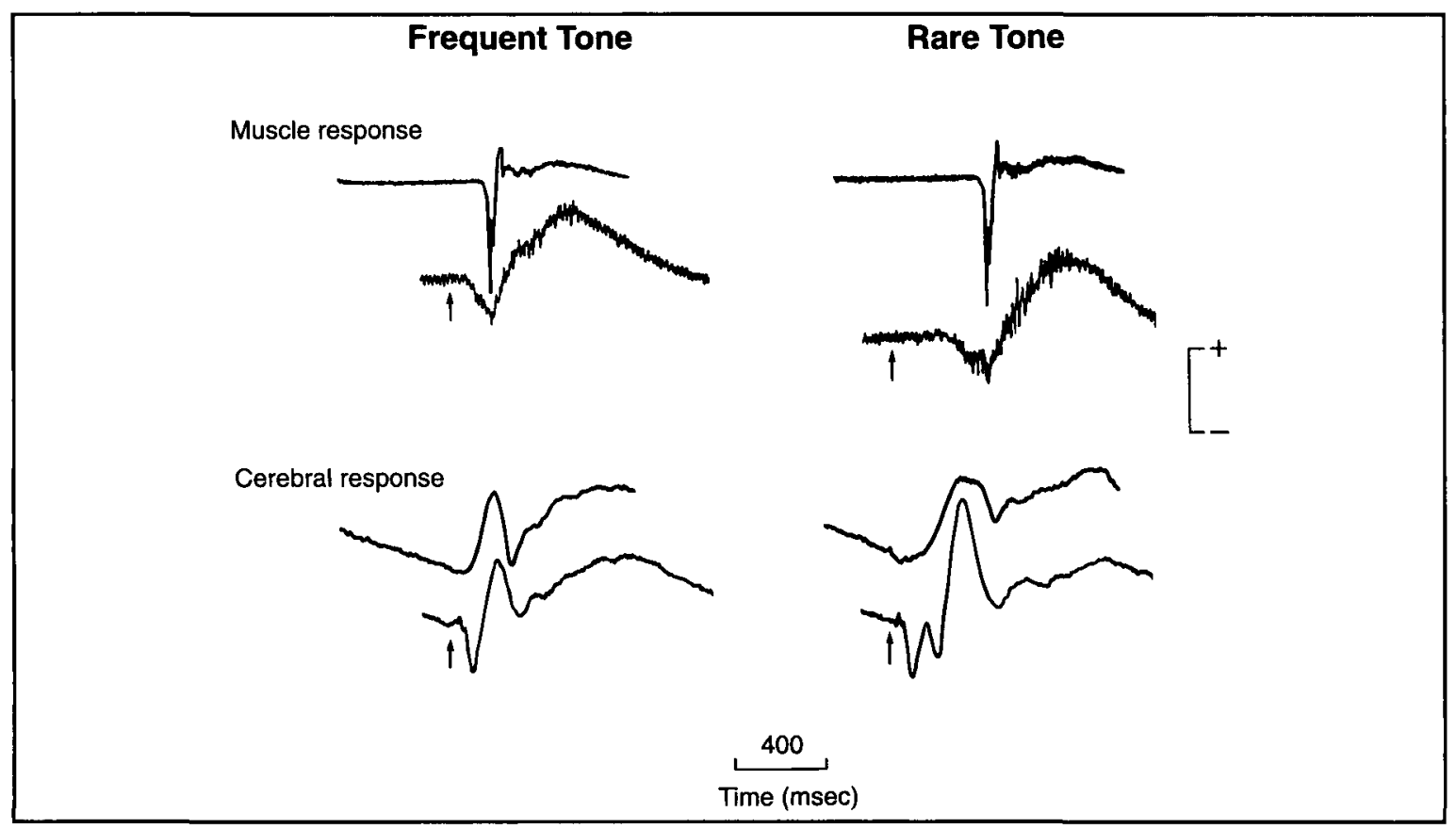

Figure 6: Response-synchronized (upper traces) and stimulus-synchronized (lower traces) grand averages of the muscle and cerebral responses to rare and frequent stimuli. Cerebral responses were recorded from $C z$ and muscle responses from the extensor digitorum communis as described earlier. Responses have been aligned by the peak of response-locked negative deflection in the averaged CMAP to facilitate identification of the cerebral components. The response to frequent tones is coupled to the P2 potential, and that to rare tones is coupled to the P3 potential. The calibration bar at the right represents $10 \mu \mathrm{V}$ for the cerebral responses, $100 \mu \mathrm{V}$ for the stimulus-synchronized muscle responses, and $200 \mu \mathrm{V}$ for the response-synchronized muscle responses. Arrow indicates stimulus onset. (Modified from Ref. 22.) 


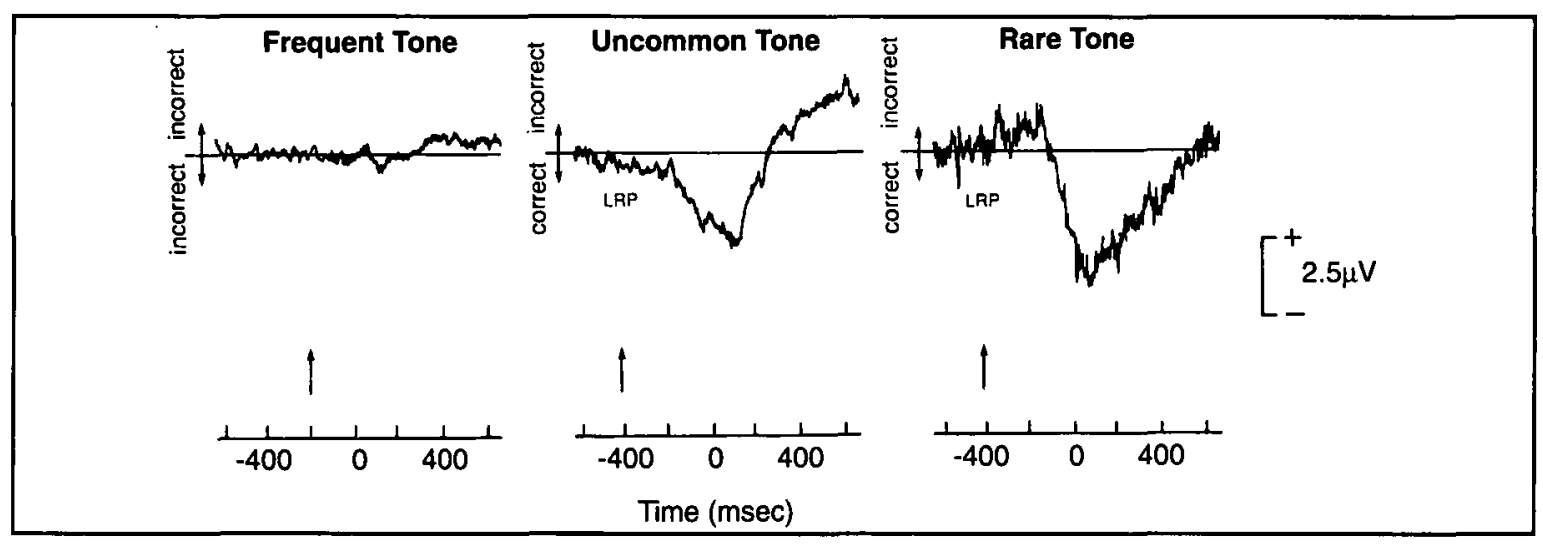

Figure 7: Grand average of the lateralized readiness potentials (LRP) preceding correct responses to the frequent, uncommon and rare tones in our three-choice experiments and calculated according to the formula:

\section{$L R P=(\Sigma[C 3-C 4]$ for right hand response $+\Sigma[C 4-C 3]$ for left hand response $) / n$}

where $n$ is the total number of responses with either hand. The LRP to the frequent tone is flat because the subject responded to this tone with both hands. The LRP to the uncommon tone is correctly lateralized contralateral to the hand that is moved. The LRP to the rare tone, by contrast, is initially lateralized incorrectly and only subsequently does the lateralization become correct. The calibration bar at the right represents $2.5 \mu V$. Arrow indicates stimulus onset. (Modified from Ref. 25.)

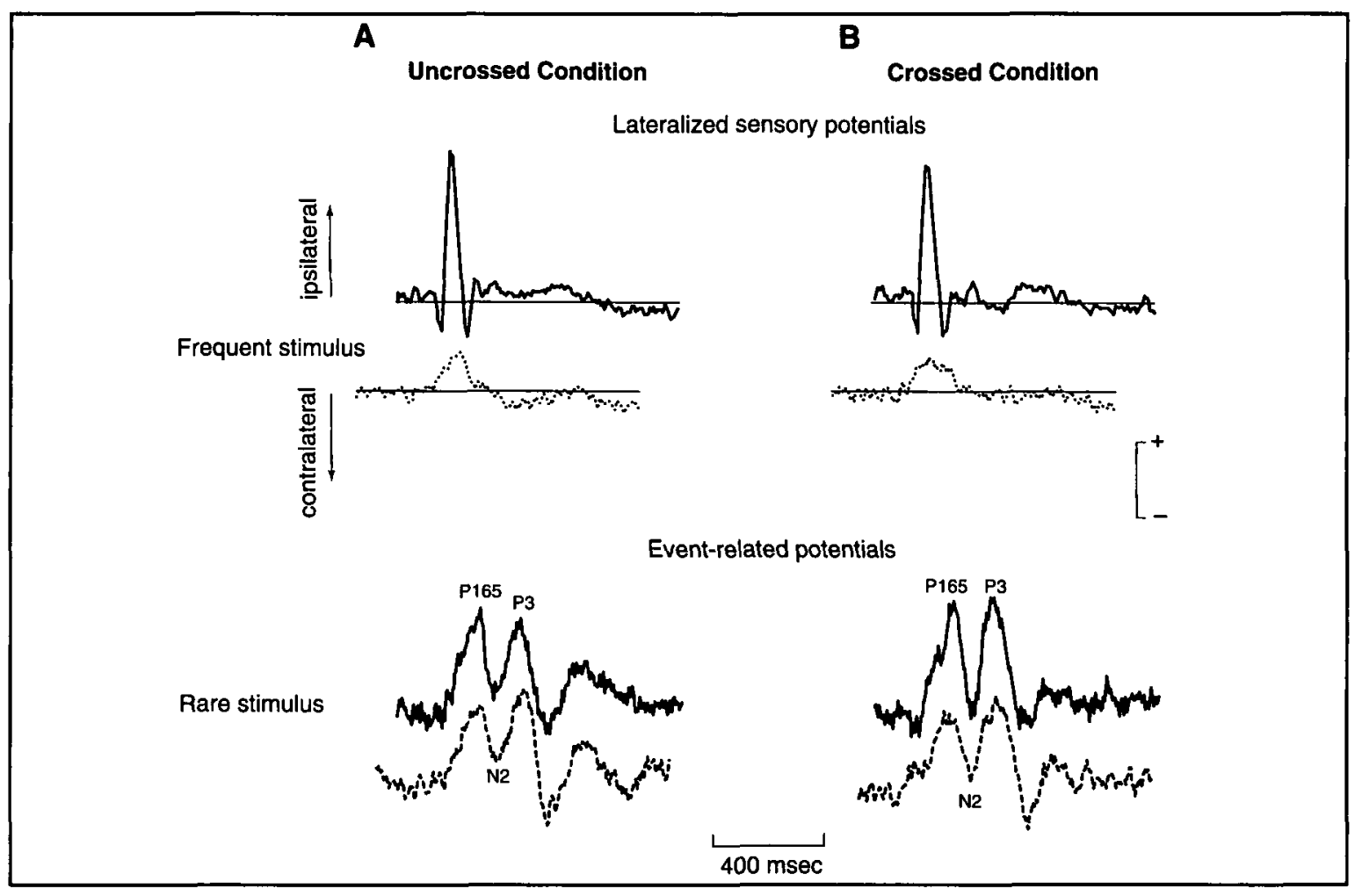

Figure 8: Grand average of the stimulus-synchronized (solid lines) and response-synchronized (dotted lines) responses to rare and frequent stimuli in our visual reaction-time experiments. The uncrossed (compatible) condition represents a response by the hand on the same side as the stimulus. The crossed (non-compatible) condition represents a response by the hand on the side opposite the stimulus. Construction of the lateralized sensory potentials (LSP) was calculated according to the formula:

$L S P=(\Sigma[P 3-P 4]$ stimulus on left $+\Sigma[P 4-P 3]$ stimulus on right $) / n$

where $n$ is the total number of stimuli to either side. The lateralized Pl00 response to the visual stimulus is markedly attenuated by response-synchronized averaging, where the entire event-related potential is preserved. The stimulus-synchronized event-related potentials depicted here represent difference waveforms obtained as described in Figure 4. The calibration bar at the right represents $2.5 \mu V$ for the lateralized sensory potential and 10 $\mu V$ for the event-related potentials. (Modified from Ref. 27.)

response-synchronized averages is not due simply to a time-locking with stimulus onset. Moreover these attenuated potentials (e.g., P100 and the error negativity) must be on a separate (i.e., parallel) branch of the stimulus processing sequence from the $\mathrm{P} 2$ and the event-related potentials that are preserved in response-synchronized averages (Figures 6 and 8 ). Presumably 


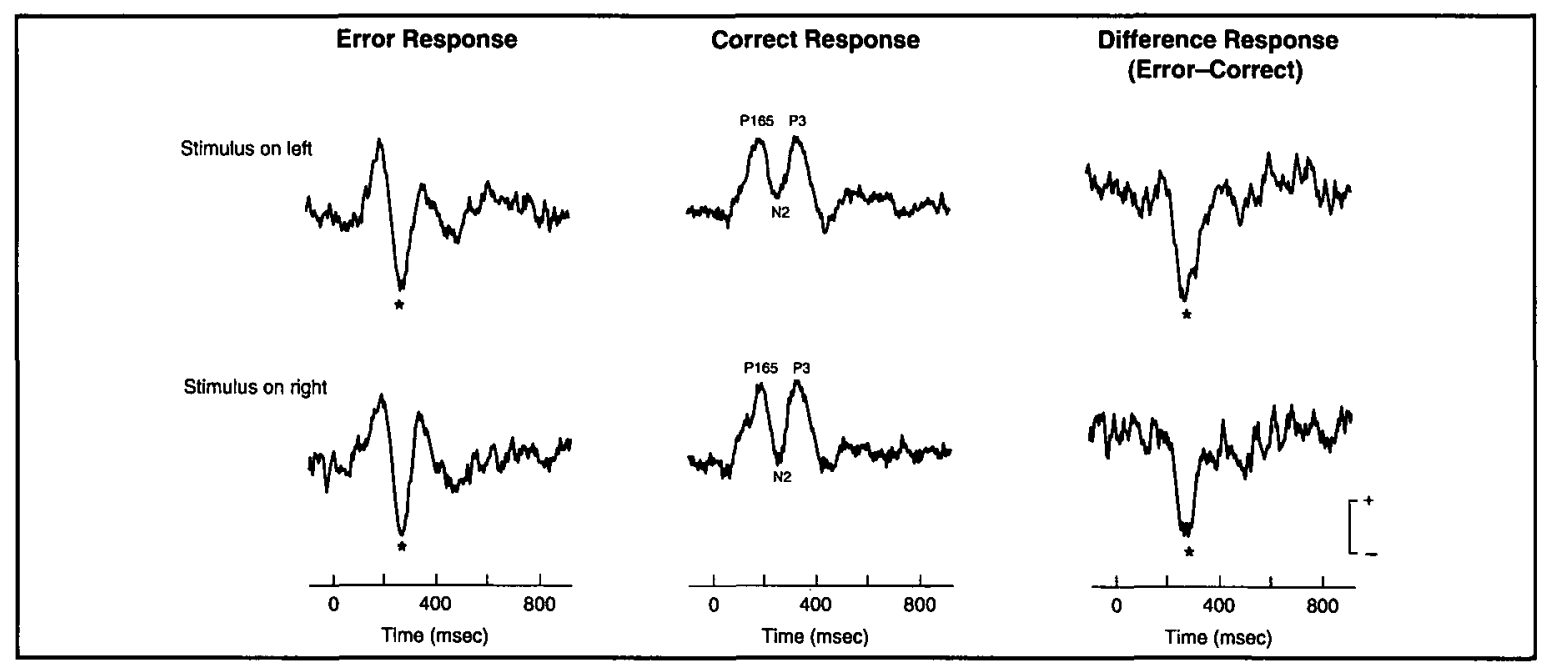

Figure 9: Grand average of the stimulus-synchronized cerebral responses for correct responses (middle traces) and error responses (left traces) in which a rare stimulus was mistaken for a frequent stimulus in our visual reaction-time experiments. Also shown (right traces) are the difference waveforms obtained by digital subtraction of the correct responses from the error responses. The main difference is the large negativity (indicated by an asterisk) that is present in the error responses and possibly reflects the subject's awareness that a mistake has been made. The calibration bar at the right represents $10 \mu \mathrm{V}$. (Modified from Ref. 27.)

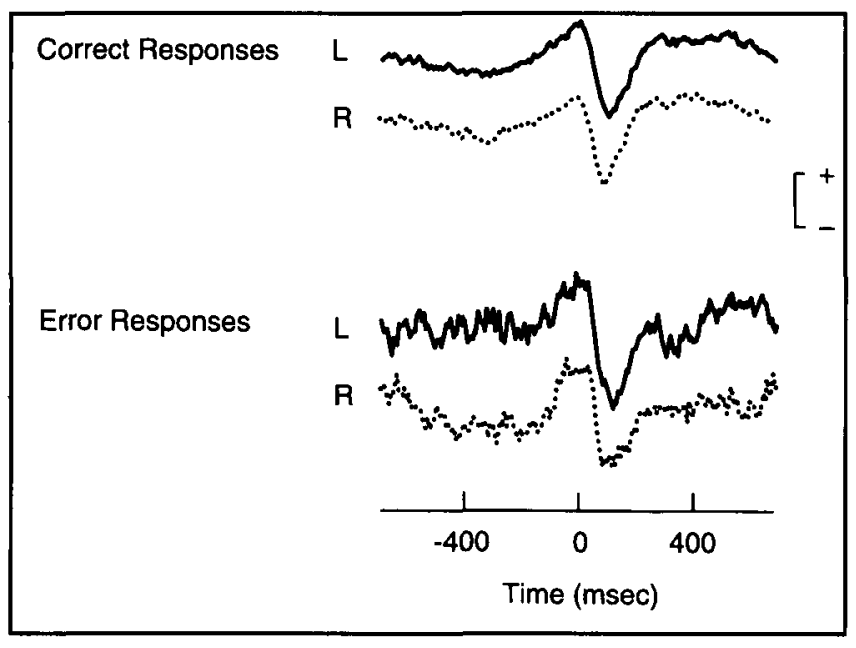

Figure 10: Grand average of the response-synchronized cerebral responses associated with correct responses to the frequent stimulus (top traces) and with error responses (bottom traces) in which a rare stimulus is mistaken for a frequent stimulus in our visual reaction time experiments. Responses to stimuli on the left are indicated by solid lines and those to stimuli on the right by dotted lines. It can be seen that the response-synchronized error responses closely resemble correct responses to the frequent stimuli and the large error negativity (Figure 9) is attenuated by the response-synchronized averaging. The calibration bar at the right represents $10 \mu \mathrm{V}$. (Modified from Ref. 27.)

the P2 and the event-related potentials (P165-N2-P3) are themselves on separate branches of the stimulus processing sequence. Three lines of evidence support this concept. First, the event-related response begins prior to and overlaps with the P2 response (Figure 2); second, the $\mathrm{P} 2$ response is not preserved in response-synchronized averages to the rare stimuli (Figure 8); and third, as discussed earlier, the event-related potential can be elicited in the absence of the N1 and P2 potentials when an anticipated stimulus is unexpectedly omitted. In this regard, it is of note that the response to the frequent stimulus is coupled to one branch (P2) whereas the response to the rare stimulus is coupled to another (P165-P3). By contrast, in our earlier threechoice experiment, both the rare and the uncommon tone evoked substantially similar event-related activity and the response to each tone was similarly coupled to this activity. ${ }^{24}$ Thus, it seems that the correct response to either infrequent tone is coupled to the same branch of the stimulus evaluation process and this may explain, in part, why the responses to the uncommon and rare tones have a similar mean latency in these experiments.

In this context, the errors made by subjects during these experiments are of interest. When subjects mistook the rare for the frequent stimulus, both the stimulus-synchronized and response-synchronized averages were altered. The stimulus-synchronized averages initially appeared similar to the averages for correct responses but the early potentials were followed by a large negativity (Figure 9). This error negativity has been found by several authors ${ }^{24,27-29}$ and possibly reflects an awareness by the subject that a mistake had been made. In the response-synchronized averages the response seemed to be coupled, not to the event-related potentials as correct responses were, but rather to the $\mathrm{P} 2$ component, as were correct responses to the frequent tone (Figure 10). Thus, it seems that the error, in this circumstance, corresponds to a coupling of the response to the wrong branch of the stimulus evaluation process.

\section{SUMMARY}

In conclusion, the results of our investigations, together with the findings of others, portray a discrimination-response system very different from the strictly serial processor envisioned by many and discussed at the outset. The picture that emerges is one of a system that is not only more complicated than a serial processor but, at the same time, more flexible and more efficient. When possible, subjects use prior knowledge (i.e., which stimulus is most probable and the likely time of its occurrence) to engage the response system in advance of stimulus onset; when such knowledge is unavailable, this anticipatory processing is lacking. The coupling of the response to the brain events 
reflected by the cerebral potentials can be altered both by the difficulty of the discrimination and by the compatibility of the response; it can even be altered by the response-strategy adopted by the subject and this strategy can be changed between trials. In addition, the responses to the frequent and rare stimuli seem to be coupled to different branches of the stimulus evaluation process and errors occur when the response is generated from the wrong branch. Indeed, the discrimination and response systems are so considerably overlapped (with variable coupling depending upon several factors), it seems implausible to expect that any specific evoked potential component will correspond to any specific stage of information processing. In fact, given the observations outlined above, even the very existence of such discrete stages must be questioned. Moreover, our results indicate clearly the existence of parallel branches of the stimulus evaluation process, and therefore, it seems probable that the cerebral evoked potentials that can be recorded during a discrimination-response task actually reflect activity in parallel neural networks rather than the activity of discrete stages of information processing. This organization presumably enables the system to be more responsive to varying circumstances perhaps, in one instance, allowing accurate responses to be generated well in advance of the full stimulus evaluation process and, in another, allowing a paradoxically rapid response to a very infrequent stimulus.

\section{REFERENCES}

1. Sutton S, Braren M, Zubin J, John ER. Evoked potential correlates of stimulus uncertainty. Science 1965; 50: 1187-1188.

2. Donchin E, Ritter W, McCallum WC. Cognitive psychophysiology: the endogenous components of the ERP. In: Calloway E, Tueting P, Koslow S, eds. Event-related Brain Potentials in Man. New York: Academic Press, 1978: 349-411.

3. Tueting P. Event-related potentials, cognitive events and information processing: a summary of issues and discussion. $I n$ : Otto D, ed. Multidisciplinary Perspectives in Event-Related Brain Potential (ERP) Research. Washington, DC: US Government Printing Office, 1978: 159-169.

4. Duncan-Johnson CC. P300 latency: a new metric of information processing. Psychophysiology 1981; 18: 207-215.

5. Hillyard SA, Kutas M. Electrophysiology of cognitive processing. Ann Rev Psychol 1983; 34: 33-61.

6. Duncan-Johnson CC, Donchin E. On quantifying surprise: the variation of event-related potentials with subjective probability. Psychophysiology 1977; 14: 456-467.

7. Squires K, Petuchowski S, Wickens C, Donchin E. The effects of stimulus sequence on event related potentials: a comparison of visual and auditory sequences. Percept Psychophys 1977; 22: 3140.

8. Squires KC, Wickens C, Squires NC, Donchin E. The effect of stimulus sequence on the waveform of the cortical event-related potential. Science 1976; 193: 1142-1146.
9. Klinke R, Fruhstorfer H, Finkenzeller P. Evoked responses as a function of external and stored information. Electroencephalogr Clin Neurophysiol 1968; 25: 119-122.

10. Picton TW, Hillyard SA. Human auditory evoked potentials-II: effects of attention. Electroencephalogr Clin Neurophysiol 1974; 36: 191199.

11. Picton TW, Hillyard SA, Krausz HI, Galambos R. Human auditory evoked potentials I: evaluation of components. Electroencephalogr Clin Neurophysiol 1974; 36: 179-190.

12. Goodin DS, Squires KC, Starr A. Variations in early and late eventrelated components of the auditory evoked potential with task difficulty. Electroencephalogr Clin Neurophysiol 1983; 55: 680-686.

13. Ritter W, Simson R, Vaughan HG, Jr. Association cortex potentials and reaction time in auditory discrimination. Electroencephalogr Clin Neurophysiol 1972; 33: 547-555.

14. Ford JM, Roth WT, Kopell BS. Auditory evoked potentials to unpredictable shifts in pitch. Psychophysiology 1976; 13: 32-39.

15. Kutas M, McCarthy G, Donchin E. Augmenting mental chronometry: the P300 as a measure of stimulus evaluation time. Science 1977; 197: 792-795.

16. McCarthy G, Donchin E. A metric for thought: a comparison of P300 latency and reaction time. Science 1981; 211: 77-80.

17. Magliero A, Bashore TR, Coles MGH, Donchin E. On the dependence of P300 latency on stimulus evaluation processes. Psychophysiology 1984; 21: 171-186.

18. Roth WT, Ford JM, Kopell BS. Long latency evoked potentials and reaction time. Psychophysiology 1978; 15: 17-23.

19. Goodin DS, Aminoff MJ. The relationship between the evoked potential and brain events in sensory discrimination and motor response. Brain 1984; 107: 241-251.

20. Goodin DS, Aminoff MJ, Mantle MM. Subclasses of event-related potentials: response-locked and stimulus-locked components. Ann Neurol 1986; 20: 603-609.

21. Goodin DS, Aminoff MJ, Mantle MM. Sensory discrimination and its relationship to the cerebral processing of infrequent stimuli. Can J Neurol Sci 1987; 14: 642-648.

22. Goodin DS, Aminoff MJ, Shefrin SL. Organization of sensory discrimination and response selection in choice and non-choice conditions: a study using cerebral evoked potentials in normal humans. J Neurophysiol 1990; 64: 1270-1281.

23. Wellford AT. Choice reaction times: basic concepts. In: Wellford AT, ed. Reaction Times. London: Academic Press, 1980: 73-128.

24. Ortiz TA, Goodin DS, Aminoff MJ. Neural processing in a threechoice reaction-time task: a study using cerebral evoked-potentials and single-trial analysis in normal humans. J Neurophysiol 1993; 69: 1499-1511.

25. Goodin DS, Aminoff MJ, Ortiz TA. Expectancy and response strategy to sensory stimuli. Neurology 1993; 43: 2139-2142.

26. Goodin DS, Aminoff MJ, Ortiz TA, Chequer RS. Response times and handedness in simple reaction-time tasks. Exp Brain Res 1996; 109: 117-126.

27. Goodin DS, Aminoff MJ, Chequer RS, Ortiz TA. Response compatibility and the relationship between event-related potentials and the timing of a motor response. J Neurophysiol 1996; 76: 3705-3713.

28. Falkenstein M, Hohnsbein J, Hoormann J, Blanke L. Effects of crossmodal divided attention on late ERP components. II. Error processing in choice reaction tasks. Electroencephalogr Clin Neurophysiol 1991; 78: 447-455.

29. Scheffers MK, Coles MGH, Bernstein P, Gehring WJ, Donchin E. Event-related brain potentials and error-related processing: an analysis of incorrect responses to go and no-go stimuli. Psychophysiology 1996; 33: 42-53. 